PROCEEDINGS OF THE

AMERICAN MATHEMATICAL SOCIETY

Volume 130, Number 1, Pages 59-67

S 0002-9939(01)06014-2

Article electronically published on May 10, 2001

\title{
ON A COMPLETE ANALYSIS \\ OF HIGH-ENERGY SCATTERING MATRIX ASYMPTOTICS FOR ONE DIMENSIONAL SCHRÖDINGER OPERATORS WITH INTEGRABLE POTENTIALS
}

\author{
ALEXEI RYBKIN \\ (Communicated by Carmen C. Chicone)
}

\begin{abstract}
For the general one dimensional Schrödinger operator $-\frac{d^{2}}{d x^{2}}+q(x)$ with real $q \in L_{1}(\mathbb{R})$ we present a complete streamlined treatment of large spectral parameter power asymptotics of Jost solutions and the scattering matrix. We find simple necessary and sufficient conditions relating the number of exact terms in the asymptotics with the smoothness of $q$. These conditions are expressed in terms of the Fourier transform of some functions related to $q$. In particular, under the usual conditions $q^{(N)} \in L_{1}(\mathbb{R}), N \in \mathbb{N}_{0}$, we derive up to two extra terms in the asymptotic expansion of the Jost solution and for the transmission coefficient we derive twice as many terms. Our main results are complete.
\end{abstract}

\section{INTRODUCTION}

For one dimensional Schrödinger operators, high-energy asymptotic expansions of the Jost solutions, Green's function, various scattering quantities, and other related functions have long been treated by both mathematicians and physicists. There is very extensive literature on this subject. We just mention a few papers containing further references: [1, [4, [5], 6], 7], 8], 9], 10], [1], 12], 13], [16].

Most of the earlier work and work done by physicists is either formal or under excessively strong conditions on potentials. However many papers mentioned are concerned with proper estimates for the remainder term that is, of course, related to the number of exact terms in the corresponding asymptotic expansions. Yet we can find only partial answers to the question on exact number of terms.

Our research in this area is, in part, inspired by a recent new interest in asymptotic representations in connection with the inverse spectral theory for scalar and matrix valued Schrödinger operators [2], [3], [8], [9], [16].

The goal of the present paper is to give a final unified exposition of various asymptotic expansions related to the Schrödinger operator. We do not claim to have new formulas and consider the work primarily methodological. Moreover we exploit

Received by the editors April 18, 2000 and, in revised form, May 15, 2000.

2000 Mathematics Subject Classification. Primary 34E05, 34L25; Secondary 34L40.

Key words and phrases. Schrödinger operator, asymptotic expansions, Jost solution, scattering matrix. 
well-known ingredients and methods but appear to have found a suitable representation of the error term. Its transparent structure immediately leads to very simple and natural necessary and sufficient conditions on the potential providing a given number of exact terms in relevant asymptotics. This lets us not only reproduce but also refine many relevant results previously obtained by others. We will comment on several improvements in the main body of the paper. Other applications to a variety of Weyl $m$-function asymptotic representations and different trace formulas will be discussed in detail elsewhere [14, [15].

\section{NOtATION AND PRELIMINARIES}

We are going to deal with the stationary Schrödinger equation on $L_{2}(\mathbb{R})$,

$$
-u^{\prime \prime}+q(x) u=z u
$$

where $z \in \mathbb{C}^{+} \equiv\{z \in \mathbb{C}: \operatorname{Im} z \geq 0\}$ and $q$ is a real locally integrable function. We agree to write $\sqrt{z} \equiv k$ if $z$ is positive. If $q \in L_{1}(\mathbb{R})$, then (2.1) has two solutions $\psi_{ \pm}(x, k)$, called Jost's, subject to the conditions $e^{\mp i k x} \psi_{ \pm}(x, k) \rightarrow 1, x \rightarrow \pm \infty$. Setting $y_{ \pm}(x, k) \equiv e^{\mp i k x} \psi_{ \pm}(x, k)$, equation (2.1) can then be represented (see e.g. [5]) as

$$
y_{ \pm}(x, k)=1+\int_{x}^{ \pm \infty} \frac{e^{2 i k(s-x)}-1}{2 i k} q(s) y_{ \pm}(s, k) d s,
$$

that implies the following asymptotic $(k>0)$ of $\psi_{ \pm}(x, k)$ as $x \rightarrow \mp \infty$ :

$$
\psi_{ \pm}(x, k)=T^{-1}(k)\left(e^{ \pm i k x}+R_{ \pm}(k) e^{\mp i k x}\right)+o(1),
$$

where $T(k)$ and $R_{ \pm}(k)$ denote the transmission and reflection coefficients from left (right) incidence. The scattering matrix $S(k)$ of the pair $\left(H, H_{0}\right)$, where $H_{0}$ $\equiv-\frac{d^{2}}{d x^{2}}, H \equiv-\frac{d^{2}}{d x^{2}}+q(x)$ are free and perturbed Schrödinger operators on $L_{2}(\mathbb{R})$, is given by

$$
S(k)=\left(\begin{array}{cc}
T(k) & R_{+}(k) \\
R_{-}(k) & T(k)
\end{array}\right), \quad k>0 .
$$

For its elements $T(k), R(k) \equiv R_{+}(k)$, and $R_{-}(k)$ one has

$$
\begin{aligned}
T^{-1}(k) & =1-\frac{1}{2 i k} \int_{\mathbb{R}} q(x) y_{+}(x, k) d x, \\
T^{-1}(k) R(k) & =\frac{1}{2 i k} \int_{\mathbb{R}} e^{2 i k x} q(x) y_{+}(x, k) d x, \\
T^{-1}(k) R_{-}(k) & =\frac{1}{2 i k} \int_{\mathbb{R}} e^{-2 i k x} q(x) y_{-}(x, k) d x .
\end{aligned}
$$

Our goal is to study the asymptotic behavior of the scattering matrix $S(k)$ as $k \rightarrow \infty$. It is enough to consider only $T(k)$ and $R(k)$ since $R_{-}(k)$ is treated analogously to $R(k)$.

We introduce the following

Definition 2.1. Let $f \in L_{1}(0, \infty)$. We call a point $x \in[0, \infty)$ an $F$-point of $f$ if there is a finite number $A(x)$ dependent only on $x$ such that

$$
\int_{0}^{\infty} e^{2 i k s} f(s+x) d s=\frac{A(x)}{2 i k}+o\left(k^{-1}\right), k \rightarrow \infty .
$$


If (2.5) holds for $k \rightarrow \infty$ inside $C_{\varepsilon} \equiv\left\{z \in \mathbb{C}^{+}: \varepsilon \leq \arg z \leq \pi-\varepsilon, \varepsilon>0\right\}$, then $x$ is called an $F C$-point of $f$.

Let $W_{1}^{n}(\Delta)$ denote the Sobolev space of integrable functions having up to $n$ distributional derivatives in $L_{1}(\Delta)$.

It is clear that if $f \in W_{1}^{1}(0, \infty)$, then any $x \in[0, \infty)$ is an $F$-point and $A(x)=$ $-f(x)$. For a discontinuous function the set of $F$-points is in general empty. In order to have a nontrivial set of $F$-points we have to impose on $f$ some smoothness. (Mere continuity is not enough.)

We will systematically be using the following elementary fact.

Lemma 2.2. (i) If $f \in W_{1}^{n}(0, \infty)$, then

$$
\int_{0}^{\infty} e^{2 i k x} f(x) d x=\sum_{j=1}^{n} \frac{(-1)^{j} f^{(j-1)}(0)}{(2 i k)^{j}}+\left(-\frac{1}{2 i k}\right)^{n} \int_{0}^{\infty} e^{2 i k x} f^{(n)}(x) d x .
$$

(ii) If $f \in W_{1}^{n}(\mathbb{R})$, then

$$
\int_{\mathbb{R}} e^{2 i k x} f(x) d x=\left(-\frac{1}{2 i k}\right)^{n} \int_{\mathbb{R}} e^{2 i k x} f^{(n)}(x) d x .
$$

Lemma 2.3. Let $f \in L_{1}(0, a), 0<a \leq \infty$. Almost every point $x \in[0, a)$ is an $F C$-point of $f$. Moreover every point $x$ subject to

$$
\int_{0}^{h}|f(x+s)-f(x)| d s=o(h), h \rightarrow+0,
$$

(called a right Lebesgue point of $f$ ) is an FC-point. For all these points $A(x)=$ $-f(x+0)$.

Lemma 2.3 is not as trivial as Lemma 2.2 and its proof can be found in [13] or derived from Theorem I.13 of [18. We are not aware of any other descriptions of $F, F C$-points.

Given a function $f(x, z), x \in \Delta \subseteq \mathbb{R}$, throughout the paper when writing $f(x, z)=\sum_{j=0}^{n} f_{j}(x) z^{-j}+o\left(|z|^{-n}\right)$ we will assume that the error term is $o\left(|z|^{-n}\right)$ uniformly in $x \in \Delta$.

\section{Asymptotics of the Jost solution}

As the title of this section suggests we are going to deal with the asymptotic expansion of the Jost solution $\psi(x, k) \equiv \psi_{+}(x, k)=e^{-i k x} y(x, k)$ where $y(x, k) \equiv$ $y_{+}(x, k)$. The function $\psi(0, k)=y(0, k)$ is commonly called the Jost function. The following proposition will play a major role in this paper.

Theorem 3.1. Let $q$ be real-valued and $q \in W_{1}^{N}(\mathbb{R})$ for some $N \in \mathbb{N}_{0} \equiv\{0,1,2, \ldots\}$. Then

(i) For almost all $x \in \mathbb{R}$,

$$
y(x, k)=1+\sum_{m=1}^{N+2} \frac{f_{m}(x)}{(2 i k)^{m}}+o\left(|k|^{-N-2}\right),|k| \rightarrow \infty, k \in C_{\varepsilon}
$$


where explicitly $\left(Q(x) \equiv \int_{x}^{\infty} q(s) d s\right)$

$$
\begin{aligned}
& f_{1}(x)=-Q(x), f_{2}(x)=-q(x)+\frac{1}{2} Q^{2}(x) \\
& f_{2}(x)=q^{\prime}(x)+q(x) Q(x)+\frac{1}{6} Q^{3}(x)+\int_{x}^{\infty} q^{2}(s) d s, \text { etc. }
\end{aligned}
$$

Moreover, all $\left\{f_{m}(x)\right\}, m=1,2, \ldots, N+1$, are continuous functions and $f_{N+2}(x)$ is defined if and only if $x$ is an FC-point of $q^{(N)}$.

(ii) For all $x \in \mathbb{R}$,

$$
y(x, k)=1+\sum_{m=1}^{N+1} \frac{f_{m}(x)}{(2 i k)^{m}}+o\left(k^{-N-1}\right), k \rightarrow \infty,
$$

i.e. on the real line we lose one term in (3.1). In order to extract the $(N+2)$-term in (3.3) at a point $x$ it is necessary and sufficient that $x$ be an F-point of $q^{(N)}$.

Proof. We first prove (ii). Assume $k>0$. Rewrite integral equation (2.2) in the form $y=1+K y$, where $K$ is a Volterra type integral operator with the kernel

$$
K(x, s)=\frac{e^{2 i k(s-x)}-1}{2 i k} q(s) .
$$

The standard iteration procedure then yields $(n \in \mathbb{N})$

$$
y=1+\sum_{m=1}^{n} K^{m} 1+K^{n+1} y
$$

Since $q \in L_{1}(\mathbb{R})$ we have

$$
\|K\| \equiv \sup _{x \in \mathbb{R}} \int_{\mathbb{R}}|K(x, s)||q(s)| d s \leq \frac{\|q\|_{1}}{k}
$$

and the iteration procedure (3.4) converges in $L_{\infty}$ to some $y$ with $\|y\|_{\infty} \leq$ $\left(1-\frac{\|q\|_{1}}{k}\right)^{-1}, k>\|q\|_{1}$, and for the error term one has

$$
\begin{aligned}
\left\|K^{n+1} y\right\|_{\infty} & \leq\|K\|^{n+1}\|y\|_{\infty} \leq\left(\frac{\|q\|_{1}}{k}\right)^{n+1}\|y\|_{\infty} \\
& \leq\left(\frac{\|q\|_{1}}{k}\right)^{n+1}\left(1-\frac{\|q\|_{1}}{k}\right)^{-1} .
\end{aligned}
$$

It follows from (3.4), 3.5 that

$$
\begin{gathered}
y(x, k)=1+\sum_{m=1}^{n} I_{m}(x, k)+o\left(k^{-n-1}\right), \\
I_{m}(k) \equiv \int_{x}^{\infty} K^{m}(x, s) d s, m \geq 2,
\end{gathered}
$$

where $K^{m}(x, s)$ are iterated kernels computed by the following recursion formulas:

$$
K^{1}(x, s)=K(x, s), \quad K^{m}(x, s)=\int_{x}^{\infty} K^{m-1}(x, t) K(t, s) d t .
$$


For $I_{m}(x, k)$ one has

$$
\begin{aligned}
I_{m}(k) & =\int_{x}^{\infty} d x_{1} \int_{x}^{x_{1}} d x_{2} K\left(x_{2}, x_{1}\right) \ldots \int_{x}^{x_{m-1}} d x_{m} K\left(x, x_{m}\right) K\left(x_{m}, x_{m-1}\right) \\
& =\left(\frac{1}{2 i k}\right)^{m}\left\{\frac{(-1)^{m} Q^{m}(x)}{m !}+\int_{0}^{\infty} e^{2 i k s} \omega_{m}(s, x) d s\right\},
\end{aligned}
$$

where explicitly $\left(Q(x) \equiv \int_{x}^{\infty} q(s) d s\right)$

$$
\begin{aligned}
& \omega_{1}(s, x)=q(s+x), \\
& \omega_{2}(s, x)=q(s+x)(Q(x)-2 Q(s+x))-\int_{x}^{\infty} q(t) q(t+s) d t, \text { etc. }
\end{aligned}
$$

We are not concerned with exact expressions for $\omega_{m}$ and what we need is that in each variable

$$
\omega_{m} \in W_{1}^{N}(\mathbb{R}), m \in \mathbb{N} .
$$

Applying Lemma 2.2 (i) with $n=N-m+2$ to $\omega_{m}(s, x), m=1,2, \ldots, N+1$, yields

$$
I_{m}(x, k)=\left(\frac{-1}{2 i k}\right)^{m}\left\{\frac{Q^{m}(x)}{m !}+\sum_{j=1}^{N-m+2} \frac{(-1)^{j-1} \omega_{m}^{(j-1)}(0, x)}{(2 i k)^{j}}\right\}+o\left(k^{-N-2}\right),
$$

and for $m=N+2$, by the Riemann-Lebesgue lemma,

$$
I_{N+2}(x, k)=\left(\frac{-1}{2 i k}\right)^{N+2} \frac{Q^{N+2}(x)}{(N+2) !}+o\left(k^{-N-2}\right) .
$$

Inserting (3.9), (3.10) into (3.6) with $n=N+2$ and combining like terms yield

$$
\begin{aligned}
y(x, k) & =\underbrace{1+\sum_{m+1}^{N+1} \frac{f_{m}(x)}{(2 i k)^{m}}}_{m=1}-\underbrace{\left(\frac{-1}{2 i k}\right)^{N+1} \int_{0}^{\infty} e^{2 i k s} q^{(N)}(s+x) d s} \\
& +\underbrace{\left(\frac{-1}{2 i k}\right)^{N+2}\left\{\frac{Q^{N+2}(x)}{(N+2) !}-\sum_{m=2}^{N+1} \omega_{m}^{(N-m+1)}(0, x)\right\}}+o\left(k^{-N-2}\right) \\
& \equiv y_{1}+y_{2}+y_{3}+o\left(k^{-N-2}\right), \quad k \rightarrow \infty,
\end{aligned}
$$

where $\left\{f_{m}(x)\right\}$ are computed by 3.2 .

We are now going to show that 3.11) implies all the required conclusions. Indeed, terms $y_{1}$ and $y_{3}$ are well defined and exact under assumption $q \in W_{1}^{N}(\mathbb{R})$ and hence the question of the exact number of terms in (3.11) depends entirely on $y_{2}$. If $q \in W_{1}^{N}(\mathbb{R})$, then, by the Riemann-Lebesgue lemma, $\int_{0}^{\infty} e^{2 i k s} q^{(N)}(s+x) d s$ $=o\left(k^{-1}\right)$ and (3.3) follows. Next, obviously we can derive the $(N+2)$-term if and only if $x$ is an $F$-point of $q^{(N)}$ and (ii) is done.

Let us now prove (i). It is well known that the Jost solution is analytic in $k$ in the upper half-plane and $y(x,-k)=\overline{y(x, k)}, k>0$. Then by the Phragmén-Lindelöf principle expansion (3.3) extends into $\mathbb{C}^{+}$. Now consider (3.11) when $|k| \rightarrow \infty, k \in$ $C_{\varepsilon}$. By Lemma $2.2 y_{2}\left(y_{2}=\left(\frac{-1}{2 i k}\right)^{N+2}(-2 i k) \int_{0}^{\infty} e^{2 i k s} q^{(N)}(s+x) d s\right)$ joints $y_{3}$ for almost all $x$ and (3.1) follows. The theorem is proven. 
Remark 3.2. Under the same condition $q \in W_{1}^{N}(\mathbb{R})$ expansion (3.3) with $N$ terms was rigorously justified in [10]. The authors conjectured on deriving the $(N+1)$ term but verified it only for $N=0$. Part (ii) of Theorem 3.1 provides an affirmative answer to their question. Moreover we explain what is needed to derive even one more term. We also notice that due to the relation

$$
m(x, z)=i \sqrt{z}+\frac{y^{\prime}(x, \sqrt{z})}{y(x, \sqrt{z})}
$$

between the Weyl $m$-function $m(x, z)$ and the Jost solution $y(x, \sqrt{z})$, Theorem 3.1 actually also implies Weyl $m$-function expansions which have drawn considerable attention (see e.g. recent papers [2, [16 and literature therein). Due to space limitation we discuss these important issues separately in [15].

Remark 3.3. In this paper we do not give an algorithm of computing coefficients $\left\{f_{m}(x)\right\}$. The interested reader can find some effective procedures in e.g. [5], [6]. There one can also find expressions for asymptotic series of the other Jost solution $\psi_{-}(x, k)$.

\section{Asymptotic expansions For the transmission AND REFLECTION COEFFICIENTS}

We now turn to the asymptotics of $T(k), R(k)$.

Theorem 4.1. Let $q$ be real-valued and $q \in W_{1}^{N}(\mathbb{R})$ for some $N \in \mathbb{N}_{0} \equiv\{0,1,2, \ldots\}$. Then

$$
T(k)=1+\sum_{m=1}^{2 N+2} \frac{t_{m}}{(2 i k)^{m}}+o\left(k^{-2 N-2}\right), k \rightarrow \infty,
$$

where explicitly $\left(Q \equiv \int_{\mathbb{R}} q(x) d x\right) \quad t_{1}=Q, t_{2}=\frac{1}{2} Q^{2}, t_{3}=-\frac{1}{6} Q^{3}-\int_{\mathbb{R}} q^{2}(x) d x$, etc.,

$$
R(k)=o\left(k^{-N-1}\right) .
$$

In order to extract the $(2 N+3)$-term in (4.1) it is necessary and sufficient that $x=0$ is an F-point of the function

$$
\int_{\mathbb{R}} q^{(N)}(s+x) q^{(N)}(s) d s .
$$

The first term in (4.2) is nontrivial if and only if the limit

$$
\lim 2 i k \cdot \int_{\mathbb{R}} e^{2 i k x} q^{(N)}(x) d x, k \rightarrow \infty,
$$

exists and differs from 0.

Proof. According to (2.3) we need to consider the following integral:

$$
J(k) \equiv \int_{\mathbb{R}} q(x) y(x, k) d x .
$$


Applying (3.6) with $n=2 N+3$ to $J(k)$ one has

$$
\begin{aligned}
J(k) & =\int_{\mathbb{R}} q(x) d x+\sum_{m=1}^{2 N+2} \int_{\mathbb{R}} q(x) I_{m}(x, k) d x+o\left(k^{-2 N-2}\right) \\
& \equiv Q+\sum_{m=1}^{2 N+2} J_{m}(k)+o\left(k^{-2 N-2}\right) .
\end{aligned}
$$

For each $J_{m}(k)$ in virtue (3.7) one has $(m \in \mathbb{N})$

$$
\begin{aligned}
J_{m}(k) & =\left(\frac{1}{2 i k}\right)^{m}\left\{\frac{(-1)^{m}}{m !} \int_{\mathbb{R}} q(x) Q^{m}(x) d x\right. \\
& \left.+\int_{\mathbb{R}} q(x)\left(\int_{0}^{\infty} e^{2 i k s} \omega_{m}(s, x) d s\right) d x\right\} .
\end{aligned}
$$

Changing the order of integration (all the functions are integrable!) in the second integral (4.4) yields

$$
\begin{aligned}
J_{m}(k) & =\left(\frac{1}{2 i k}\right)^{m} \frac{(-1)^{m+1}}{(m+1) !} Q^{m+1} \\
& +\left(\frac{1}{2 i k}\right)^{m} \int_{0}^{\infty} e^{2 i k x}\left(\int_{\mathbb{R}} q(s) \omega_{m}(x, s) d s\right) d x .
\end{aligned}
$$

Set $g_{m}(x) \equiv \int_{\mathbb{R}} q(s) \omega_{m}(x, s) d s$. We claim that $g_{m} \in W_{1}^{2 N}(\mathbb{R})$. Due to space limitation we prove it only for $m=1,2$. In force of (3.8), in the distributional sense one can easily justify the following chain of differentiation:

$$
\begin{aligned}
& g_{1}^{(2 N)}(x)=\left(\int_{\mathbb{R}} q(s) q^{\prime}(s+x) d s\right)^{(2 N-1)}=-\left(\int_{\mathbb{R}} q^{\prime}(s) q(s+x) d s\right)^{(2 N-1)} \\
& =\ldots=(-1)^{N}\left(\int_{\mathbb{R}} q^{(N)}(s) q(s+x) d s\right)^{(N)}=(-1)^{N} \int_{\mathbb{R}} q^{(N)}(s) q^{(N)}(s+x) d s .
\end{aligned}
$$

Clearly $\left\|g_{1}^{(2 N)}\right\|_{1} \leq\left\|q^{(N)}\right\|_{1}^{2}$ and we conclude that $g_{1} \in W_{1}^{2 N}(\mathbb{R})$. For $g_{2}^{(2 N)}$, similar to (4.6) one can easily get

$$
\begin{aligned}
g_{2}^{(2 N)}(x) & =(-1)^{N}\left\{2 \int_{\mathbb{R}}(q(s) Q(s))^{(N)} q^{(N)}(s+x) d s\right. \\
& \left.-Q \int_{\mathbb{R}} q^{(N)}(s) q^{(N)}(s+x) d s-2 \int_{\mathbb{R}} q^{(N)}(s)(q(s+x) Q(s+x))^{(N)} d s\right\},
\end{aligned}
$$

that immediately implies

$$
\left\|g_{2}^{(2 N)}\right\|_{1} \leq 3\left\|(q Q)^{(N)}\right\|_{1}\left\|q^{(N)}\right\|_{1}+|Q|\left\|q^{(N)}\right\|_{1}^{2}
$$

and hence $g_{2} \in W_{1}^{2 N}(\mathbb{R})$. Now applying Lemma 2.2 (i) with $n=2 N$ to $g_{m}$ one has $(m \in \mathbb{N})$

$$
\int_{0}^{\infty} e^{2 i k x} g_{m}(x) d x=\sum_{j=1}^{2 N} \frac{(-1)^{j} g_{m}^{(j-1)}(0)}{(2 i k)^{j}}+\left(\frac{1}{2 i k}\right)^{2 N} \int_{0}^{\infty} e^{2 i k x} g_{m}^{(2 N)}(x) d x
$$


Combining it with (4.4) we finally obtain

$$
\begin{aligned}
J_{m}(k) & =\left(\frac{1}{2 i k}\right)^{m}\left\{\frac{(-1)^{m+1}}{(m+1) !} Q^{m+1}+\sum_{j=1}^{2 N} \frac{(-1)^{j} g_{m}^{(j-1)}(0)}{(2 i k)^{j}}\right. \\
& \left.+\left(\frac{1}{2 i k}\right)^{2 N} \cdot \int_{0}^{\infty} e^{2 i k x} g_{m}^{(2 N)}(x) d x\right\} .
\end{aligned}
$$

Let us control the terms in $J_{m}(k)$ up to order $2 N+2$

$$
\begin{gathered}
J_{m}(k)=\left(\frac{1}{2 i k}\right)^{m}\left\{\frac{(-1)^{m+1}}{(m+1) !} Q^{m+1}+\sum_{j=1}^{2 N-m+2} \frac{(-1)^{j} g_{m}^{(j-1)}(0)}{(2 i k)^{j}}\right\} \\
+o\left(k^{-2 N-2}\right), m=1,2, \ldots, 2 N+1, \\
J_{2 N+2}(k)=\left(\frac{1}{2 i k}\right)^{2 N+2} \frac{-1}{(2 N+3) !} Q^{2 N+3}+o\left(k^{-2 N-2}\right) .
\end{gathered}
$$

Combining (2.3), (4.3) and (4.7) we finally arrive at

$$
\begin{aligned}
& T^{-1}(k)=\left\{1+\sum_{m=1}^{2 N+2} \frac{a_{m}}{(2 i k)^{m}}\right\}-\left(\frac{1}{2 i k}\right)^{2 N+2} \int_{0}^{\infty} e^{2 i k x} g_{1}^{(2 N)}(x) d x \\
& +\left(\frac{1}{2 i k}\right)^{2 N+3}\left\{\frac{-Q^{2 N+3}}{(2 N+3) !}+\sum_{m=2}^{2 N+1}(-1)^{m} g_{m}^{(2 N-m+1)}(0)\right\}+o\left(k^{-2 N-3}\right),
\end{aligned}
$$

where explicitly $a_{1}=-Q, a_{2}=-\frac{1}{2} Q^{2}, a_{3}=\frac{1}{6} Q^{2}+\int_{\mathbb{R}} q^{2}(x) d x$, etc. Observe that (4.8) has a structure similar to (3.11). Applying similar arguments we have proven the part of the theorem related to $T(k)$.

Turn now to the reflection coefficient $R(k)$. Inserting (3.3) into (2.4) we have

$$
\begin{aligned}
& T^{-1}(k) R(k)=\frac{1}{2 i k} \int_{\mathbb{R}} e^{2 i k x} q(x) y(x, k) d x=\frac{1}{2 i k} \int_{\mathbb{R}} e^{2 i k x} q(x) d x \\
& +\sum_{m=2}^{N+1} \frac{1}{(2 i k)^{m}} \int_{\mathbb{R}} e^{2 i k x} f_{m}(x) d x+o\left(k^{-N-1}\right) .
\end{aligned}
$$

Thus by Lemma 2.2 (ii), (4.9) reads

$$
T^{-1}(k) R(k)=(-1)^{N}\left(\frac{1}{2 i k}\right)^{N+1} \int_{\mathbb{R}} e^{2 i k x} q^{(N)}(x) d x+o\left(k^{-N-2}\right)
$$

and one can easily complete the proof.

Remark 4.2. The asymptotic expansion (4.1) looks quite surprising since it has been generally believed (see, e.g. [5], 10]) that the condition $q \in W_{1}^{N}(\mathbb{R})$, roughly speaking, allows for $N$ terms in (4.1). In [10] the $(N+1)$-term was conjectured; we double this number under the same condition. (4.2) was proven in [10] in the general case and also in [7] for $N=2$.

Remark 4.3. One can easily see that the condition $q^{(N)} \in L_{2}(\mathbb{R})$ is necessary for extracting the $(2 N+3)$-term in (4.1). 


\section{REFERENCES}

1. V.S. Buslaev and L.D. Faddeev, "Formulas for traces for a singular Sturm-Liouville differential operator", Soviet Math. Dokl. 1 (1960), 451-454. MR 22:11171

2. S. Clark and F. Gesztesy, "Weyl-Titchmarsh $M$-function asymptotics for matrix-valued Schrödinger operators", Proc. London Math. Soc., CMP 20001:02

3. S. Clark, F. Gesztesy, H. Holden, and B. Levitan, "Borg-type theorems for matrix-valued Schrödinger operators", J. Diff. Eq. 167 (2000), 181-210. CMP 2001:02

4. L.D. Faddeev and V.E. Zakharov, "Korteweg-De Vries equation: a completely integrable Hamiltonian system", Funct. Anal. Appl. 5, 4 (1971), 280-287.

5. F. Gesztesy and H. Holden, "Trace formulas and conservation laws for nonlinear evolution equation", Rev. Math. Phys. 6, 1 (1994), 51-95. MR 95h:35198a errata MR 95h:35198b

6. F. Gesztesy, H. Holden, B. Simon, and Z. Zhao, "Higher order trace relations for Schrödinger operators", Rev. Math. Phys. 7, 6 (1995), 893-922. MR 97d:34094

7. F. Gesztesy, H. Holden, and B. Simon, "Absolute summability of the trace relation for certain Schrödinger operators", Commun. Math. Phys. 168 (1995), 137-161. MR 96b:34110

8. F. Gesztesy and B. Simon, "A new approach to inverse spectral theory, II. General real potentials and the connection to the spectral measure", Ann. Math. 152 (2000), 593-643. CMP 2001:06

9. F. Gesztesy and B. Simon, "On local Borg-Marchenko uniqueness results", Comm. Math. Phys. 211 (2000), 273-287. MR 2001b:34020

10. D.B. Hinton, M. Klaus and J.K. Shaw, "High-energy asymptotics of the scattering matrix on the line", Inverse Problems 5 (1989), 1049-1056. MR 91f:34107

11. D.B. Hinton, M. Klaus and J.K. Shaw, "Series representation and asymptotics for of the Titchmarsh-Weyl $m$-functions", Differential Integral Equations 2, 4 (1989), 420-429. MR 90m:34057

12. T. Miyazawa, "Boson representations of one-dimensional scattering", J. Phys. A: Math. Gen. 33 (2000), 191-225. CMP 2000:10

13. A.V. Rybkin, "On the trace approach to the inverse scattering in dimension one", SIAM J. Math. Anal. 32 (2001), no 6, 1248-1264.

14. A.V. Rybkin, "A Weyl $m$-function approach to trace formulas for varies Schrödinger operators", in preparation.

15. A.V. Rybkin, "Some new and old asymptotic representations of the Jost solution and Weyl $m$-function for Schrödinger operators on the line", to appear in Bulletin of LMS.

16. B. Simon, "A new approach to inverse spectral theory, I. Fundamental formalism", Annals of Math. 150 (1999), 1029-1057. CMP 2000:08

17. E.C. Titchmarsh, "On eigenfunction expansions associated with second-order differential equations", Oxford University Press, 1950.

18. E.C. Titchmarsh, "Introduction to the theory of Fourier integrals", Chelsea, New York, 1986. MR 89c:42002

Department of Mathematical Sciences, University of Alaska-Fairbanks, P.O. Box 756660, Fairbanks, Alaska 99775

E-mail address: ffavr@uaf.edu 